Acta Crystallographica Section E

Structure Reports

Online

ISSN 1600-5368

\section{Bis[benzyl $N^{\prime}$-(1H-indol-3-ylmethylene)- hydrazinecarbodithioato- $\left.\kappa^{2} N^{\prime}, S\right]-$ nickel(II) $N, N$-dimethylformamide disolvate}

\author{
Hamid Khaledi, Hapipah Mohd Ali and Seik Weng Ng* \\ Department of Chemistry, University of Malaya, 50603 Kuala Lumpur, Malaysia \\ Correspondence e-mail: seikweng@um.edu.my
}

Received 15 November 2008; accepted 19 November 2008

Key indicators: single-crystal X-ray study; $T=100 \mathrm{~K}$; mean $\sigma(\mathrm{C}-\mathrm{C})=0.004 \AA$;

$R$ factor $=0.040 ; w R$ factor $=0.094 ;$ data-to-parameter ratio $=13.8$.

In the title compound, $\left[\mathrm{Ni}\left(\mathrm{C}_{17} \mathrm{H}_{14} \mathrm{~N}_{3} \mathrm{~S}_{2}\right)_{2}\right] \cdot 2 \mathrm{C}_{3} \mathrm{H}_{7} \mathrm{NO}$, the $\mathrm{Ni}$ atom (site symmetry $\overline{1}$ ) is $N, S$-chelated by two deprotonated Schiff base anions in a distorted square-planar geometry. The dihedral angle between the aromatic ring planes within the ligand is $86.37(13)^{\circ}$. In the crystal structure, an $\mathrm{N}-\mathrm{H} \cdots \mathrm{O}$ hydrogen bond links the complex to the dimethylformamide solvent molecule.

\section{Related literature}

For other square-planar nickel dithiocarbazates, see: Ali et al. (2000); Tian et al. (1996a,b); Xue et al. (2003); Zhang et al. (2004); Zhu et al. (2000).

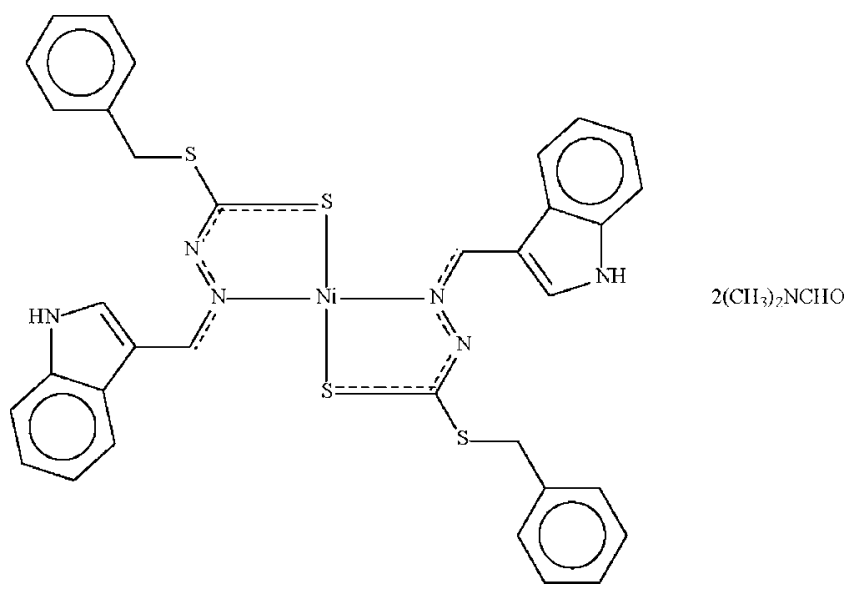

Monoclinic, $P 2_{1} / c$

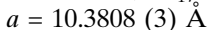

$b=20.0219$ (7) $\AA$

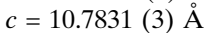

$\beta=117.921(2)^{\circ}$

$V=1980.3(1) \AA^{3}$

Data collection

Bruker SMART APEX CCD diffractometer

Absorption correction: multi-scan (SADABS; Sheldrick, 1996) $T_{\min }=0.916, T_{\max }=0.957$

Refinement

$R\left[F^{2}>2 \sigma\left(F^{2}\right)\right]=0.040$

$w R\left(F^{2}\right)=0.094$

$S=1.03$

3481 reflections
$Z=2$

Mo $K \alpha$ radiation $\mu=0.75 \mathrm{~mm}^{-1}$ $T=100$ (2) K

$0.12 \times 0.12 \times 0.06 \mathrm{~mm}$

13342 measured reflections 3481 independent reflections 2615 reflections with $I>2 \sigma(I)$ $R_{\text {int }}=0.062$

Table 1

Selected bond lengths (A).

\begin{tabular}{llll}
\hline $\mathrm{Ni1}-\mathrm{N} 2$ & $1.916(2)$ & $\mathrm{Ni1}-\mathrm{S} 1$ & $2.1770(7)$ \\
\hline
\end{tabular}

Data collection: APEX2 (Bruker, 2007); cell refinement: SAINT (Bruker, 2007); data reduction: $S A I N T$; $\operatorname{program}(\mathrm{s})$ used to solve structure: SHELXS97 (Sheldrick, 2008); program(s) used to refine structure: SHELXL97 (Sheldrick, 2008); molecular graphics: $X$ SEED (Barbour, 2001); software used to prepare material for publication: pubCIF (Westrip, 2008).

We thank the University of Malaya for funding this study (Science Fund grants 12-02-03-2031, 12-02-03-2051).

Supplementary data and figures for this paper are available from the IUCr electronic archives (Reference: HB2849).

\title{
References
}

Ali, M. A., Mirza, A., Butcher, R. J. \& Rahman, M. (2000). Transition Met. Chem. 25, 430-436.

Barbour, L. J. (2001). J. Supramol. Chem. 1, 189-191.

Bruker (2007). APEX2 and SAINT. Bruker AXS Inc., Madison, Wisconsin, USA.

Sheldrick, G. M. (1996). SADABS. University of Göttingen, Germany.

Sheldrick, G. M. (2008). Acta Cryst. A64, 112-122.

Tian, Y.-P., Duan, C.-Y., Lu, Z.-L., You, X.-Z., Fun, H.-K. \& Kandasamy, S. (1996a). Polyhedron, 15, 2263-2271.

Tian, Y.-P., Duan, C.-Y., Lu, Z.-L., You, X.-Z. \& Huang, X.-Y. (1996b). J. Coord. Chem. 38, 219-226.

Westrip, S. P. (2008). publCIF. In preparation.

Xue, Z.-M., Zhang, X.-J., Tian, Y.-P., Wu, J.-Y., Jiang, M.-H. \& Fun, H.-K. (2003). Chin. J. Struct. Chem. 22, 265-269.

Zhang, M.-L., Tian, Y.-P., Zhang, X.-J., Wu, J.-Y., Zhang, S.-Y., Wang, D., Jiang, M.-H., Chantrapromma, S. \& Fun, H.-K. (2004). Transition Met. Chem. 29, 596-602.

Zhu, X.-H., Chen, X.-F., Zhang, Y., You, X.-Z., Tan, W.-L., Ji, W., Vittal, J. J., Tan, G.-K. \& Kennard, C. H. L. (2000). New J. Chem. 24, 419-423.

\section{Experimental}

Crystal data

$\left[\mathrm{Ni}\left(\mathrm{C}_{17} \mathrm{H}_{14} \mathrm{~N}_{3} \mathrm{~S}_{2}\right)_{2}\right] \cdot 2 \mathrm{C}_{3} \mathrm{H}_{7} \mathrm{NO} \quad M_{r}=853.77$ 


\section{supporting information}

Acta Cryst. (2008). E64, m1615 [doi:10.1107/S1600536808038580]

\section{Bis[benzyl $N^{\prime}$-(1 H-indol-3-ylmethylene)hydrazinecarbodithioato- $\left.\kappa^{2} N^{\prime}, S\right]$ nickel(II) $N, N$-dimethylformamide disolvate}

\section{Hamid Khaledi, Hapipah Mohd Ali and Seik Weng Ng}

\section{S1. Comment}

For related structures, see: Ali et al. (2000); Tian et al. (1996a,b); Xue et al. (2003); Zhang et al. (2004); Zhu et al. (2000).

\section{S2. Experimental}

Benzyl (1H-indol-2-ylmethylene)hydrazinecarbodithioate ethanol hemisolvate ( $2 \mathrm{mmol}, 0.65 \mathrm{~g})$ was dissolved in ethanol $(30 \mathrm{ml})$ along with several drops of triethylamine. To the resulting clear solution was added an ethanol solution (10 ml) containing $1 \mathrm{mmol}(0.25 \mathrm{~g})$ of nickel acetate tetrahydrate. The mixture was heated for an hour. The product that separated was recrystallized from DMF to yield brown blocks of (I).

\section{S3. Refinement}

Hydrogen atoms were placed at calculated positions $(\mathrm{C}-\mathrm{H}=0.95-0.99 \AA, \mathrm{N}-\mathrm{H}=0.88 \AA)$ and refined as riding with $U(\mathrm{H})$ $=1.2-1.5$ times $U_{\text {eq }}(C, N)$.

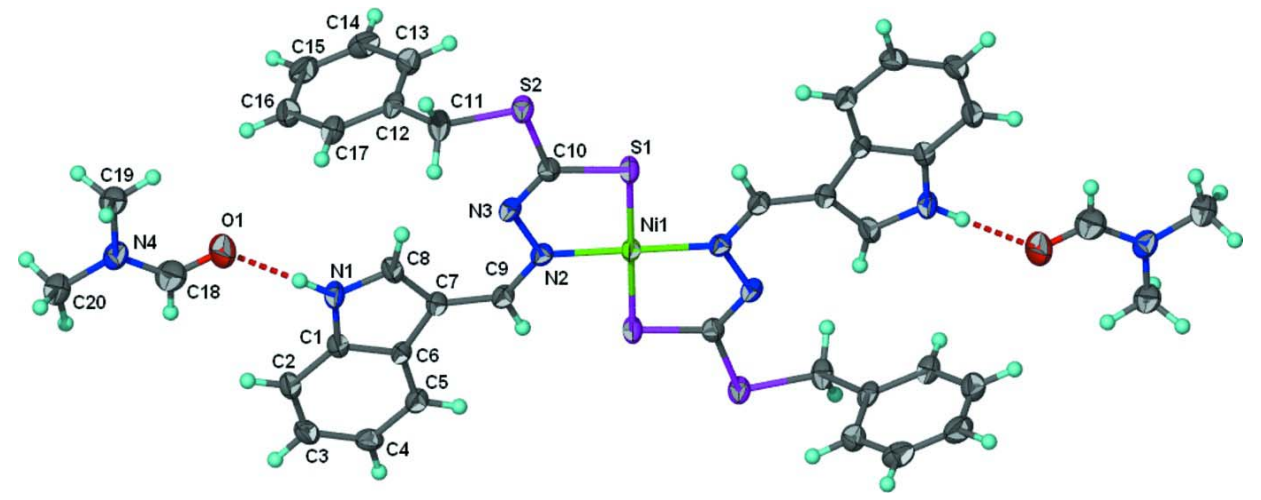

Figure 1

View of (I) at the $70 \%$ probability level. Hydrogen atoms are drawn as spheres of arbitrary radius. The unlabelled atoms are generated by the symmetry operation $(1-\mathrm{x}, 1-\mathrm{y}, 1-\mathrm{z})$ and the hydrogen bonds are shown as dashed lines.

Bis[benzyl $N^{\prime}$-(1H-indol-3-ylmethylene)hydrazinecarbodithioato- $\left.\kappa^{2} N^{\prime}, S\right]$ nickel(II) $N, N$-dimethylformamide disolvate

Crystal data

$\left[\mathrm{Ni}\left(\mathrm{C}_{17} \mathrm{H}_{14} \mathrm{~N}_{3} \mathrm{~S}_{2}\right)_{2}\right] \cdot 2 \mathrm{C}_{3} \mathrm{H}_{7} \mathrm{NO} \quad$ Monoclinic, $P 2_{1} / c$

$M_{r}=853.77 \quad$ Hall symbol: $-\mathrm{P} 2 \mathrm{ybc}$ 
$a=10.3808$ (3) $\AA$

$b=20.0219$ (7) $\AA$

$c=10.7831(3) \AA$

$\beta=117.921(2)^{\circ}$

$V=1980.3(1) \AA^{3}$

$Z=2$

$F(000)=892$

$D_{\mathrm{x}}=1.432 \mathrm{Mg} \mathrm{m}^{-3}$

Data collection

Bruker SMART APEX CCD

diffractometer

Radiation source: fine-focus sealed tube

Graphite monochromator

$\omega$ scans

Absorption correction: multi-scan

(SADABS; Sheldrick, 1996)

$T_{\min }=0.916, T_{\max }=0.957$

Refinement

Refinement on $F^{2}$

Least-squares matrix: full

$R\left[F^{2}>2 \sigma\left(F^{2}\right)\right]=0.040$

$w R\left(F^{2}\right)=0.094$

$S=1.03$

3481 reflections

252 parameters

0 restraints

Primary atom site location: structure-invariant direct methods
Mo $K \alpha$ radiation, $\lambda=0.71073 \AA$

Cell parameters from 1450 reflections

$\theta=2.2-22.7^{\circ}$

$\mu=0.75 \mathrm{~mm}^{-1}$

$T=100 \mathrm{~K}$

Block, brown

$0.12 \times 0.12 \times 0.06 \mathrm{~mm}$

13342 measured reflections

3481 independent reflections

2615 reflections with $I>2 \sigma(I)$

$R_{\text {int }}=0.062$

$\theta_{\text {max }}=25.0^{\circ}, \theta_{\min }=2.0^{\circ}$

$h=-12 \rightarrow 12$

$k=-23 \rightarrow 23$

$l=-12 \rightarrow 12$

Secondary atom site location: difference Fourier map

Hydrogen site location: inferred from neighbouring sites

$\mathrm{H}$-atom parameters constrained

$w=1 /\left[\sigma^{2}\left(F_{\mathrm{o}}^{2}\right)+(0.0396 P)^{2}+0.2888 P\right]$

where $P=\left(F_{\mathrm{o}}^{2}+2 F_{\mathrm{c}}^{2}\right) / 3$

$(\Delta / \sigma)_{\max }=0.001$

$\Delta \rho_{\max }=0.50 \mathrm{e} \AA^{-3}$

$\Delta \rho_{\min }=-0.29 \mathrm{e} \AA^{-3}$

Fractional atomic coordinates and isotropic or equivalent isotropic displacement parameters $\left(\hat{A}^{2}\right)$

\begin{tabular}{lllll}
\hline & $x$ & $y$ & $z$ & $U_{\mathrm{iso}}^{*} / U_{\mathrm{eq}}$ \\
\hline Ni1 & 0.5000 & 0.5000 & 0.5000 & $0.01710(15)$ \\
S1 & $0.48640(8)$ & $0.60841(4)$ & $0.48343(8)$ & $0.0236(2)$ \\
S2 & $0.38426(8)$ & $0.70431(4)$ & $0.62163(8)$ & $0.0234(2)$ \\
O1 & $0.1755(3)$ & $0.56685(12)$ & $1.1091(2)$ & $0.0472(7)$ \\
N1 & $0.2818(3)$ & $0.49406(11)$ & $0.9618(2)$ & $0.0220(6)$ \\
H1 & 0.2520 & 0.5191 & 1.0105 & $0.026^{*}$ \\
N2 & $0.4331(2)$ & $0.51020(11)$ & $0.6371(2)$ & $0.0182(5)$ \\
N3 & $0.3953(2)$ & $0.57357(11)$ & $0.6685(2)$ & $0.0192(5)$ \\
N4 & $0.0849(3)$ & $0.57529(12)$ & $1.2634(2)$ & $0.0252(6)$ \\
C1 & $0.2942(3)$ & $0.42563(14)$ & $0.9707(3)$ & $0.0192(6)$ \\
C2 & $0.2658(3)$ & $0.38131(15)$ & $1.0548(3)$ & $0.0232(7)$ \\
H2 & 0.2351 & 0.3967 & 1.1200 & $0.028^{*}$ \\
C3 & $0.2842(3)$ & $0.31463(15)$ & $1.0393(3)$ & $0.0260(7)$ \\
H3 & 0.2647 & 0.2832 & 1.0945 & $0.031^{*}$ \\
C4 & $0.3308(3)$ & $0.29162(15)$ & $0.9445(3)$ & $0.0250(7)$ \\
H4 & 0.3412 & 0.2450 & 0.9354 & $0.030^{*}$ \\
C5 & $0.3621(3)$ & $0.33626(15)$ & $0.8636(3)$ & $0.0219(7)$ \\
H5 & 0.3955 & 0.3205 & 0.8005 & $0.026^{*}$
\end{tabular}




$\begin{array}{lllll}\text { C6 } & 0.3437(3) & 0.40438(14) & 0.8763(3) & 0.0187(6) \\ \text { C7 } & 0.3625(3) & 0.46401(14) & 0.8099(3) & 0.0188(6) \\ \text { C8 } & 0.3220(3) & 0.51697(15) & 0.8678(3) & 0.0223(7) \\ \text { H8 } & 0.3227 & 0.5627 & 0.8443 & 0.027^{*} \\ \text { C9 } & 0.4138(3) & 0.46239(15) & 0.7091(3) & 0.0198(7) \\ \text { H9 } & 0.4382 & 0.4191 & 0.6907 & 0.024^{*} \\ \text { C10 } & 0.4193(3) & 0.62076(14) & 0.6009(3) & 0.0191(6) \\ \text { C11 } & 0.3353(3) & 0.70343(15) & 0.7620(3) & 0.0245(7) \\ \text { H11A } & 0.4028 & 0.6726 & 0.8354 & 0.029^{*} \\ \text { H11B } & 0.3535 & 0.7487 & 0.8039 & 0.029^{*} \\ \text { C12 } & 0.1817(3) & 0.68378(14) & 0.7277(3) & 0.0206(7) \\ \text { C13 } & 0.0673(3) & 0.68232(15) & 0.5927(3) & 0.0259(7) \\ \text { H13 } & 0.0838 & 0.6939 & 0.5159 & 0.031^{*} \\ \text { C14 } & -0.0716(3) & 0.66398(16) & 0.5687(3) & 0.0309(8) \\ \text { H14 } & -0.1490 & 0.6622 & 0.4754 & 0.037^{*} \\ \text { C15 } & -0.0977(3) & 0.64846(16) & 0.6793(3) & 0.0320(8) \\ \text { H15 } & -0.1930 & 0.6364 & 0.6627 & 0.038^{*} \\ \text { C16 } & 0.0152(3) & 0.65049(15) & 0.8139(3) & 0.0291(8) \\ \text { H16 } & -0.0020 & 0.6398 & 0.8908 & 0.035^{*} \\ \text { C17 } & 0.1540(3) & 0.66816(15) & 0.8379(3) & 0.0243(7) \\ \text { H17 } & 0.2312 & 0.6696 & 0.9313 & 0.029^{*} \\ \text { C18 } & 0.1381(4) & 0.54196(18) & 1.1919(3) & 0.0376(9) \\ \text { H18 } & 0.1485 & 0.4950 & 1.2054 & 0.045^{*} \\ \text { C19 } & 0.0701(3) & 0.64730(15) & 1.2501(3) & 0.0289(8) \\ \text { H19A } & 0.0755 & 0.6616 & 1.1658 & 0.043^{*} \\ \text { H19B } & 0.1489 & 0.6684 & 1.3329 & 0.043^{*} \\ \text { H19C } & -0.0241 & 0.6606 & 1.2427 & 0.043^{*} \\ \text { C20 } & 0.0490(3) & 0.54232(15) & 1.3634(3) & 0.0293(7) \\ \text { H20A } & 0.0590 & 0.4939 & 1.3579 & 0.044^{*} \\ \text { H20B } & -0.0516 & 0.5531 & 1.3412 & 0.044^{*} \\ \text { H20C } & 0.1154 & 0.5578 & 1.4584 & 0.044^{*}\end{array}$

Atomic displacement parameters $\left(\AA^{2}\right)$

\begin{tabular}{lllllll}
\hline & $U^{11}$ & $U^{22}$ & $U^{33}$ & $U^{12}$ & $U^{13}$ & $U^{23}$ \\
\hline Ni1 & $0.0178(3)$ & $0.0178(3)$ & $0.0192(3)$ & $-0.0001(2)$ & $0.0116(2)$ & $-0.0001(2)$ \\
S1 & $0.0326(5)$ & $0.0197(4)$ & $0.0292(4)$ & $0.0005(3)$ & $0.0234(4)$ & $0.0002(3)$ \\
S2 & $0.0297(5)$ & $0.0183(4)$ & $0.0296(4)$ & $-0.0010(3)$ & $0.0201(4)$ & $-0.0016(3)$ \\
O1 & $0.0752(19)$ & $0.0443(17)$ & $0.0405(14)$ & $0.0204(13)$ & $0.0424(14)$ & $0.0058(12)$ \\
N1 & $0.0274(14)$ & $0.0212(15)$ & $0.0242(13)$ & $-0.0007(11)$ & $0.0178(11)$ & $-0.0028(11)$ \\
N2 & $0.0186(13)$ & $0.0152(14)$ & $0.0220(12)$ & $0.0001(10)$ & $0.0106(10)$ & $-0.0005(10)$ \\
N3 & $0.0197(13)$ & $0.0175(14)$ & $0.0223(13)$ & $0.0009(10)$ & $0.0114(11)$ & $-0.0031(10)$ \\
N4 & $0.0265(15)$ & $0.0263(16)$ & $0.0239(13)$ & $0.0015(11)$ & $0.0129(12)$ & $-0.0056(11)$ \\
C1 & $0.0192(16)$ & $0.0213(17)$ & $0.0180(14)$ & $-0.0012(13)$ & $0.0094(12)$ & $0.0003(12)$ \\
C2 & $0.0225(17)$ & $0.0289(19)$ & $0.0212(15)$ & $-0.0016(14)$ & $0.0128(13)$ & $0.0023(13)$ \\
C3 & $0.0232(17)$ & $0.030(2)$ & $0.0264(16)$ & $0.0009(14)$ & $0.0128(14)$ & $0.0100(14)$ \\
C4 & $0.0229(17)$ & $0.0197(17)$ & $0.0319(17)$ & $0.0021(13)$ & $0.0123(14)$ & $0.0079(13)$ \\
C5 & $0.0188(16)$ & $0.0253(18)$ & $0.0229(15)$ & $0.0023(13)$ & $0.0110(13)$ & $0.0003(13)$
\end{tabular}


supporting information

\begin{tabular}{lllllll} 
C6 & $0.0129(15)$ & $0.0237(18)$ & $0.0205(15)$ & $0.0019(12)$ & $0.0088(12)$ & $0.0023(12)$ \\
C7 & $0.0172(16)$ & $0.0200(17)$ & $0.0203(15)$ & $-0.0010(12)$ & $0.0098(13)$ & $-0.0001(12)$ \\
C8 & $0.0243(17)$ & $0.0242(18)$ & $0.0217(15)$ & $-0.0019(13)$ & $0.0134(13)$ & $0.0033(12)$ \\
C9 & $0.0172(16)$ & $0.0201(18)$ & $0.0237(16)$ & $0.0008(12)$ & $0.0107(13)$ & $-0.0002(12)$ \\
C10 & $0.0162(16)$ & $0.0222(17)$ & $0.0183(14)$ & $-0.0012(12)$ & $0.0076(12)$ & $-0.0008(12)$ \\
C11 & $0.0304(18)$ & $0.0232(18)$ & $0.0263(16)$ & $-0.0054(14)$ & $0.0186(14)$ & $-0.0074(13)$ \\
C12 & $0.0251(17)$ & $0.0148(16)$ & $0.0282(16)$ & $0.0002(13)$ & $0.0177(14)$ & $-0.0055(12)$ \\
C13 & $0.0301(19)$ & $0.0247(18)$ & $0.0265(17)$ & $0.0025(14)$ & $0.0164(15)$ & $-0.0007(13)$ \\
C14 & $0.0224(18)$ & $0.032(2)$ & $0.0314(18)$ & $0.0053(14)$ & $0.0071(15)$ & $-0.0008(15)$ \\
C15 & $0.0232(18)$ & $0.031(2)$ & $0.044(2)$ & $0.0045(14)$ & $0.0177(16)$ & $0.0048(15)$ \\
C16 & $0.0322(19)$ & $0.0273(19)$ & $0.0366(19)$ & $0.0018(14)$ & $0.0234(16)$ & $0.0042(14)$ \\
C17 & $0.0246(18)$ & $0.0256(18)$ & $0.0264(16)$ & $-0.0007(14)$ & $0.0150(14)$ & $-0.0043(13)$ \\
C18 & $0.048(2)$ & $0.034(2)$ & $0.0333(19)$ & $0.0081(17)$ & $0.0206(18)$ & $-0.0021(16)$ \\
C19 & $0.034(2)$ & $0.028(2)$ & $0.0260(17)$ & $0.0037(15)$ & $0.0150(15)$ & $-0.0037(14)$ \\
C20 & $0.0300(18)$ & $0.029(2)$ & $0.0324(18)$ & $-0.0026(14)$ & $0.0177(15)$ & $-0.0049(14)$ \\
\hline
\end{tabular}

Geometric parameters $\left(\hat{A},{ }^{o}\right)$

\begin{tabular}{|c|c|c|c|}
\hline $\mathrm{Ni} 1-\mathrm{N} 2^{\mathrm{i}}$ & $1.916(2)$ & $\mathrm{C} 6-\mathrm{C} 7$ & $1.451(4)$ \\
\hline $\mathrm{N} 11-\mathrm{N} 2$ & $1.916(2)$ & $\mathrm{C} 7-\mathrm{C} 8$ & $1.391(4)$ \\
\hline $\mathrm{Ni1}-\mathrm{S} 1$ & $2.1770(7)$ & $\mathrm{C} 7-\mathrm{C} 9$ & $1.418(4)$ \\
\hline $\mathrm{Ni1}-\mathrm{S} 1^{\mathrm{i}}$ & $2.1770(7)$ & $\mathrm{C} 8-\mathrm{H} 8$ & 0.9500 \\
\hline $\mathrm{S} 1-\mathrm{C} 10$ & $1.725(3)$ & $\mathrm{C} 9-\mathrm{H} 9$ & 0.9500 \\
\hline $\mathrm{S} 2-\mathrm{C} 10$ & $1.748(3)$ & $\mathrm{C} 11-\mathrm{C} 12$ & $1.511(4)$ \\
\hline $\mathrm{S} 2-\mathrm{C} 11$ & $1.808(3)$ & $\mathrm{C} 11-\mathrm{H} 11 \mathrm{~A}$ & 0.9900 \\
\hline $\mathrm{O} 1-\mathrm{C} 18$ & $1.233(4)$ & C11-H11B & 0.9900 \\
\hline $\mathrm{N} 1-\mathrm{C} 8$ & $1.346(3)$ & $\mathrm{C} 12-\mathrm{C} 17$ & $1.383(4)$ \\
\hline $\mathrm{N} 1-\mathrm{C} 1$ & $1.375(3)$ & $\mathrm{C} 12-\mathrm{C} 13$ & $1.382(4)$ \\
\hline $\mathrm{N} 1-\mathrm{H} 1$ & 0.8800 & $\mathrm{C} 13-\mathrm{C} 14$ & $1.390(4)$ \\
\hline $\mathrm{N} 2-\mathrm{C} 9$ & $1.306(3)$ & $\mathrm{C} 13-\mathrm{H} 13$ & 0.9500 \\
\hline $\mathrm{N} 2-\mathrm{N} 3$ & $1.416(3)$ & $\mathrm{C} 14-\mathrm{C} 15$ & $1.377(4)$ \\
\hline $\mathrm{N} 3-\mathrm{C} 10$ & $1.287(3)$ & $\mathrm{C} 14-\mathrm{H} 14$ & 0.9500 \\
\hline $\mathrm{N} 4-\mathrm{C} 18$ & $1.321(4)$ & $\mathrm{C} 15-\mathrm{C} 16$ & $1.375(4)$ \\
\hline $\mathrm{N} 4-\mathrm{C} 20$ & $1.454(4)$ & $\mathrm{C} 15-\mathrm{H} 15$ & 0.9500 \\
\hline $\mathrm{N} 4-\mathrm{C} 19$ & $1.450(4)$ & $\mathrm{C} 16-\mathrm{C} 17$ & $1.385(4)$ \\
\hline $\mathrm{C} 1-\mathrm{C} 2$ & $1.395(4)$ & $\mathrm{C} 16-\mathrm{H} 16$ & 0.9500 \\
\hline $\mathrm{C} 1-\mathrm{C} 6$ & $1.404(4)$ & $\mathrm{C} 17-\mathrm{H} 17$ & 0.9500 \\
\hline $\mathrm{C} 2-\mathrm{C} 3$ & $1.370(4)$ & $\mathrm{C} 18-\mathrm{H} 18$ & 0.9500 \\
\hline $\mathrm{C} 2-\mathrm{H} 2$ & 0.9500 & $\mathrm{C} 19-\mathrm{H} 19 \mathrm{~A}$ & 0.9800 \\
\hline $\mathrm{C} 3-\mathrm{C} 4$ & $1.398(4)$ & C19-H19B & 0.9800 \\
\hline $\mathrm{C} 3-\mathrm{H} 3$ & 0.9500 & $\mathrm{C} 19-\mathrm{H} 19 \mathrm{C}$ & 0.9800 \\
\hline $\mathrm{C} 4-\mathrm{C} 5$ & $1.390(4)$ & $\mathrm{C} 20-\mathrm{H} 20 \mathrm{~A}$ & 0.9800 \\
\hline $\mathrm{C} 4-\mathrm{H} 4$ & 0.9500 & $\mathrm{C} 20-\mathrm{H} 20 \mathrm{~B}$ & 0.9800 \\
\hline $\mathrm{C} 5-\mathrm{C} 6$ & $1.393(4)$ & $\mathrm{C} 20-\mathrm{H} 20 \mathrm{C}$ & 0.9800 \\
\hline $\mathrm{C} 5-\mathrm{H} 5$ & 0.9500 & & \\
\hline $\mathrm{N} 2 \mathrm{i}-\mathrm{N} 11-\mathrm{N} 2$ & 180.0 & $\mathrm{C} 7-\mathrm{C} 9-\mathrm{H} 9$ & 114.5 \\
\hline $\mathrm{N} 2 \mathrm{i}-\mathrm{Ni} 1-\mathrm{S} 1$ & $94.36(7)$ & $\mathrm{N} 3-\mathrm{C} 10-\mathrm{S} 1$ & $124.2(2)$ \\
\hline
\end{tabular}




\begin{tabular}{|c|c|c|c|}
\hline $\mathrm{N} 2-\mathrm{Ni1}-\mathrm{S} 1$ & $85.64(7)$ & $\mathrm{N} 3-\mathrm{C} 10-\mathrm{S} 2$ & $121.5(2)$ \\
\hline $\mathrm{N} 22^{\mathrm{i}}-\mathrm{Ni} 1-\mathrm{S} 1^{\mathrm{i}}$ & $85.64(7)$ & $\mathrm{S} 1-\mathrm{C} 10-\mathrm{S} 2$ & $114.26(16)$ \\
\hline $\mathrm{N} 2-\mathrm{Ni} 1-\mathrm{S} 1^{\mathrm{i}}$ & $94.36(7)$ & $\mathrm{C} 12-\mathrm{C} 11-\mathrm{S} 2$ & $118.4(2)$ \\
\hline $\mathrm{S} 1-\mathrm{Ni} 1-\mathrm{S} 1^{\mathrm{i}}$ & 180.0 & $\mathrm{C} 12-\mathrm{C} 11-\mathrm{H} 11 \mathrm{~A}$ & 107.7 \\
\hline $\mathrm{C} 10-\mathrm{S} 1-\mathrm{Ni} 1$ & $96.64(10)$ & $\mathrm{S} 2-\mathrm{C} 11-\mathrm{H} 11 \mathrm{~A}$ & 107.7 \\
\hline $\mathrm{C} 10-\mathrm{S} 2-\mathrm{C} 11$ & $104.76(13)$ & $\mathrm{C} 12-\mathrm{C} 11-\mathrm{H} 11 \mathrm{~B}$ & 107.7 \\
\hline $\mathrm{C} 8-\mathrm{N} 1-\mathrm{C} 1$ & $109.9(2)$ & $\mathrm{S} 2-\mathrm{C} 11-\mathrm{H} 11 \mathrm{~B}$ & 107.7 \\
\hline $\mathrm{C} 8-\mathrm{N} 1-\mathrm{H} 1$ & 125.1 & $\mathrm{H} 11 \mathrm{~A}-\mathrm{C} 11-\mathrm{H} 11 \mathrm{~B}$ & 107.1 \\
\hline $\mathrm{C} 1-\mathrm{N} 1-\mathrm{H} 1$ & 125.1 & $\mathrm{C} 17-\mathrm{C} 12-\mathrm{C} 13$ & $118.5(3)$ \\
\hline $\mathrm{C} 9-\mathrm{N} 2-\mathrm{N} 3$ & $112.2(2)$ & $\mathrm{C} 17-\mathrm{C} 12-\mathrm{C} 11$ & $118.0(2)$ \\
\hline $\mathrm{C} 9-\mathrm{N} 2-\mathrm{Ni} 1$ & $126.4(2)$ & $\mathrm{C} 13-\mathrm{C} 12-\mathrm{C} 11$ & $123.5(3)$ \\
\hline $\mathrm{N} 3-\mathrm{N} 2-\mathrm{Ni} 1$ & $121.46(17)$ & $\mathrm{C} 12-\mathrm{C} 13-\mathrm{C} 14$ & $120.4(3)$ \\
\hline $\mathrm{C} 10-\mathrm{N} 3-\mathrm{N} 2$ & $111.9(2)$ & $\mathrm{C} 12-\mathrm{C} 13-\mathrm{H} 13$ & 119.8 \\
\hline $\mathrm{C} 18-\mathrm{N} 4-\mathrm{C} 20$ & $121.8(3)$ & $\mathrm{C} 14-\mathrm{C} 13-\mathrm{H} 13$ & 119.8 \\
\hline $\mathrm{C} 18-\mathrm{N} 4-\mathrm{C} 19$ & $119.9(3)$ & $\mathrm{C} 15-\mathrm{C} 14-\mathrm{C} 13$ & $120.4(3)$ \\
\hline $\mathrm{C} 20-\mathrm{N} 4-\mathrm{C} 19$ & $118.2(2)$ & $\mathrm{C} 15-\mathrm{C} 14-\mathrm{H} 14$ & 119.8 \\
\hline $\mathrm{N} 1-\mathrm{C} 1-\mathrm{C} 2$ & $129.6(3)$ & $\mathrm{C} 13-\mathrm{C} 14-\mathrm{H} 14$ & 119.8 \\
\hline $\mathrm{N} 1-\mathrm{C} 1-\mathrm{C} 6$ & $107.8(2)$ & $\mathrm{C} 14-\mathrm{C} 15-\mathrm{C} 16$ & $119.4(3)$ \\
\hline $\mathrm{C} 2-\mathrm{C} 1-\mathrm{C} 6$ & $122.6(3)$ & $\mathrm{C} 14-\mathrm{C} 15-\mathrm{H} 15$ & 120.3 \\
\hline $\mathrm{C} 3-\mathrm{C} 2-\mathrm{C} 1$ & $117.2(3)$ & $\mathrm{C} 16-\mathrm{C} 15-\mathrm{H} 15$ & 120.3 \\
\hline $\mathrm{C} 3-\mathrm{C} 2-\mathrm{H} 2$ & 121.4 & $\mathrm{C} 15-\mathrm{C} 16-\mathrm{C} 17$ & $120.2(3)$ \\
\hline $\mathrm{C} 1-\mathrm{C} 2-\mathrm{H} 2$ & 121.4 & $\mathrm{C} 15-\mathrm{C} 16-\mathrm{H} 16$ & 119.9 \\
\hline $\mathrm{C} 2-\mathrm{C} 3-\mathrm{C} 4$ & $121.7(3)$ & $\mathrm{C} 17-\mathrm{C} 16-\mathrm{H} 16$ & 119.9 \\
\hline $\mathrm{C} 2-\mathrm{C} 3-\mathrm{H} 3$ & 119.2 & $\mathrm{C} 12-\mathrm{C} 17-\mathrm{C} 16$ & $121.0(3)$ \\
\hline $\mathrm{C} 4-\mathrm{C} 3-\mathrm{H} 3$ & 119.2 & $\mathrm{C} 12-\mathrm{C} 17-\mathrm{H} 17$ & 119.5 \\
\hline $\mathrm{C} 5-\mathrm{C} 4-\mathrm{C} 3$ & $120.7(3)$ & $\mathrm{C} 16-\mathrm{C} 17-\mathrm{H} 17$ & 119.5 \\
\hline $\mathrm{C} 5-\mathrm{C} 4-\mathrm{H} 4$ & 119.7 & $\mathrm{O} 1-\mathrm{C} 18-\mathrm{N} 4$ & $125.3(3)$ \\
\hline $\mathrm{C} 3-\mathrm{C} 4-\mathrm{H} 4$ & 119.7 & $\mathrm{O} 1-\mathrm{C} 18-\mathrm{H} 18$ & 117.3 \\
\hline $\mathrm{C} 4-\mathrm{C} 5-\mathrm{C} 6$ & 119.0 & $\mathrm{~N} 4-\mathrm{C} 18-\mathrm{H} 18$ & 117.3 \\
\hline $\mathrm{C} 4-\mathrm{C} 5-\mathrm{H} 5$ & 120.5 & N4-C19-H19A & 109.5 \\
\hline $\mathrm{C} 6-\mathrm{C} 5-\mathrm{H} 5$ & 120.5 & N4-C19-H19B & 109.5 \\
\hline $\mathrm{C} 5-\mathrm{C} 6-\mathrm{C} 1$ & $118.8(3)$ & $\mathrm{H} 19 \mathrm{~A}-\mathrm{C} 19-\mathrm{H} 19 \mathrm{~B}$ & 109.5 \\
\hline $\mathrm{C} 5-\mathrm{C} 6-\mathrm{C} 7$ & $134.5(3)$ & N4-C19-H19C & 109.5 \\
\hline $\mathrm{C} 1-\mathrm{C} 6-\mathrm{C} 7$ & $106.7(2)$ & $\mathrm{H} 19 \mathrm{~A}-\mathrm{C} 19-\mathrm{H} 19 \mathrm{C}$ & 109.5 \\
\hline $\mathrm{C} 8-\mathrm{C} 7-\mathrm{C} 9$ & $131.5(3)$ & $\mathrm{H} 19 \mathrm{~B}-\mathrm{C} 19-\mathrm{H} 19 \mathrm{C}$ & 109.5 \\
\hline $\mathrm{C} 8-\mathrm{C} 7-\mathrm{C} 6$ & $105.5(2)$ & $\mathrm{N} 4-\mathrm{C} 20-\mathrm{H} 20 \mathrm{~A}$ & 109.5 \\
\hline $\mathrm{C} 9-\mathrm{C} 7-\mathrm{C} 6$ & $123.0(3)$ & $\mathrm{N} 4-\mathrm{C} 20-\mathrm{H} 20 \mathrm{~B}$ & 109.5 \\
\hline $\mathrm{N} 1-\mathrm{C} 8-\mathrm{C} 7$ & $110.1(3)$ & $\mathrm{H} 20 \mathrm{~A}-\mathrm{C} 20-\mathrm{H} 20 \mathrm{~B}$ & 109.5 \\
\hline $\mathrm{N} 1-\mathrm{C} 8-\mathrm{H} 8$ & 125.0 & $\mathrm{~N} 4-\mathrm{C} 20-\mathrm{H} 20 \mathrm{C}$ & 109.5 \\
\hline $\mathrm{C} 7-\mathrm{C} 8-\mathrm{H} 8$ & 125.0 & $\mathrm{H} 20 \mathrm{~A}-\mathrm{C} 20-\mathrm{H} 20 \mathrm{C}$ & 109.5 \\
\hline $\mathrm{N} 2-\mathrm{C} 9-\mathrm{C} 7$ & $131.1(3)$ & $\mathrm{H} 20 \mathrm{~B}-\mathrm{C} 20-\mathrm{H} 20 \mathrm{C}$ & 109.5 \\
\hline $\mathrm{N} 2-\mathrm{C} 9-\mathrm{H} 9$ & 114.5 & & \\
\hline $\mathrm{N} 2{ }^{\mathrm{i}}-\mathrm{N} i 1-\mathrm{S} 1-\mathrm{C} 10$ & $177.84(11)$ & $\mathrm{C} 9-\mathrm{C} 7-\mathrm{C} 8-\mathrm{N} 1$ & $-179.2(3)$ \\
\hline $\mathrm{N} 2-\mathrm{Ni} 1-\mathrm{S} 1-\mathrm{C} 10$ & $-2.16(11)$ & $\mathrm{C} 6-\mathrm{C} 7-\mathrm{C} 8-\mathrm{N} 1$ & $0.2(3)$ \\
\hline $\mathrm{S} 1-\mathrm{Ni} 1-\mathrm{N} 2-\mathrm{C} 9$ & $-177.9(2)$ & $\mathrm{N} 3-\mathrm{N} 2-\mathrm{C} 9-\mathrm{C} 7$ & $0.1(4)$ \\
\hline $\mathrm{S} 1{ }^{\mathrm{i}}-\mathrm{Ni} 1-\mathrm{N} 2-\mathrm{C} 9$ & $2.1(2)$ & $\mathrm{Ni} 1-\mathrm{N} 2-\mathrm{C} 9-\mathrm{C} 7$ & $-178.5(2)$ \\
\hline $\mathrm{S} 1-\mathrm{Ni} 1-\mathrm{N} 2-\mathrm{N} 3$ & $3.52(18)$ & $\mathrm{C} 8-\mathrm{C} 7-\mathrm{C} 9-\mathrm{N} 2$ & $-2.8(5)$ \\
\hline
\end{tabular}




$\begin{array}{ll}\mathrm{S} 1-\mathrm{i}-\mathrm{Ni} 1-\mathrm{N} 2-\mathrm{N} 3 & -176.48(18) \\ \mathrm{C} 9-\mathrm{N} 2-\mathrm{N} 3-\mathrm{C} 10 & 177.8(2) \\ \mathrm{N} 11-\mathrm{N} 2-\mathrm{N} 3-\mathrm{C} 10 & -3.5(3) \\ \mathrm{C} 8-\mathrm{N} 1-\mathrm{C} 1-\mathrm{C} 2 & 179.3(3) \\ \mathrm{C} 8-\mathrm{N} 1-\mathrm{C} 1-\mathrm{C} 6 & -0.5(3) \\ \mathrm{N} 1-\mathrm{C} 1-\mathrm{C} 2-\mathrm{C} 3 & 178.4(3) \\ \mathrm{C} 6-\mathrm{C} 1-\mathrm{C} 2-\mathrm{C} 3 & -1.9(4) \\ \mathrm{C} 1-\mathrm{C} 2-\mathrm{C} 3-\mathrm{C} 4 & 0.7(4) \\ \mathrm{C} 2-\mathrm{C} 3-\mathrm{C} 4-\mathrm{C} 5 & 0.8(4) \\ \mathrm{C} 3-\mathrm{C} 4-\mathrm{C} 5-\mathrm{C} 6 & -1.2(4) \\ \mathrm{C} 4-\mathrm{C} 5-\mathrm{C} 6-\mathrm{C} 1 & 0.0(4) \\ \mathrm{C} 4-\mathrm{C} 5-\mathrm{C} 6-\mathrm{C} 7 & -178.9(3) \\ \mathrm{N} 1-\mathrm{C} 1-\mathrm{C} 6-\mathrm{C} 5 & -178.6(2) \\ \mathrm{C} 2-\mathrm{C} 1-\mathrm{C} 6-\mathrm{C} 5 & 1.5(4) \\ \mathrm{N} 1-\mathrm{C} 1-\mathrm{C} 6-\mathrm{C} 7 & 0.6(3) \\ \mathrm{C} 2-\mathrm{C} 1-\mathrm{C} 6-\mathrm{C} 7 & -179.3(3) \\ \mathrm{C} 5-\mathrm{C} 6-\mathrm{C} 7-\mathrm{C} 8 & 178.6(3) \\ \mathrm{C} 1-\mathrm{C} 6-\mathrm{C} 7-\mathrm{C} 8 & -0.5(3) \\ \mathrm{C} 5-\mathrm{C} 6-\mathrm{C} 7-\mathrm{C} 9 & -2.0(5) \\ \mathrm{C} 1-\mathrm{C} 6-\mathrm{C} 7-\mathrm{C} 9 & 179.0(2) \\ \mathrm{C} 1-\mathrm{N} 1-\mathrm{C} 8-\mathrm{C} 7 & 0.2(3)\end{array}$

$$
\begin{aligned}
& \mathrm{C} 6-\mathrm{C} 7-\mathrm{C} 9-\mathrm{N} 2 \\
& \mathrm{~N} 2-\mathrm{N} 3-\mathrm{C} 10-\mathrm{S} 1 \\
& \mathrm{~N} 2-\mathrm{N} 3-\mathrm{C} 10-\mathrm{S} 2 \\
& \mathrm{~N} 1-\mathrm{S} 1-\mathrm{C} 10-\mathrm{N} 3 \\
& \mathrm{~N} 1-\mathrm{S} 1-\mathrm{C} 10-\mathrm{S} 2 \\
& \mathrm{C} 11-\mathrm{S} 2-\mathrm{C} 10-\mathrm{N} 3 \\
& \mathrm{C} 11-\mathrm{S} 2-\mathrm{C} 10-\mathrm{S} 1 \\
& \mathrm{C} 10-\mathrm{S} 2-\mathrm{C} 11-\mathrm{C} 12 \\
& \mathrm{~S} 2-\mathrm{C} 11-\mathrm{C} 12-\mathrm{C} 17 \\
& \mathrm{~S} 2-\mathrm{C} 11-\mathrm{C} 12-\mathrm{C} 13 \\
& \mathrm{C} 17-\mathrm{C} 12-\mathrm{C} 13-\mathrm{C} 14 \\
& \mathrm{C} 11-\mathrm{C} 12-\mathrm{C} 13-\mathrm{C} 14 \\
& \mathrm{C} 12-\mathrm{C} 13-\mathrm{C} 14-\mathrm{C} 15 \\
& \mathrm{C} 13-\mathrm{C} 14-\mathrm{C} 15-\mathrm{C} 16 \\
& \mathrm{C} 14-\mathrm{C} 15-\mathrm{C} 16-\mathrm{C} 17 \\
& \mathrm{C} 13-\mathrm{C} 12-\mathrm{C} 17-\mathrm{C} 16 \\
& \mathrm{C} 11-\mathrm{C} 12-\mathrm{C} 17-\mathrm{C} 16 \\
& \mathrm{C} 15-\mathrm{C} 16-\mathrm{C} 17-\mathrm{C} 12 \\
& \mathrm{C} 20-\mathrm{N} 4-\mathrm{C} 18-\mathrm{O} 1 \\
& \mathrm{C} 19-\mathrm{N} 4-\mathrm{C} 18-\mathrm{O} 1
\end{aligned}
$$

$177.9(3)$

1.1 (3)

$-179.25(17)$

1.2 (2)

$-178.47(13)$

6.4 (3)

$-173.95(15)$

-80.3 (2)

$165.3(2)$

$-16.5(4)$

-1.5 (4)

$-179.7(3)$

$1.4(5)$

-0.7 (5)

$0.1(5)$

0.9 (4)

$179.2(3)$

-0.2 (5)

$-177.4(3)$

$-1.6(5)$

Symmetry code: (i) $-x+1,-y+1,-z+1$.

Hydrogen-bond geometry $\left(A,{ }^{\circ}\right)$

\begin{tabular}{lllll}
\hline$D-\mathrm{H} \cdots A$ & $D-\mathrm{H}$ & $\mathrm{H} \cdots A$ & $D \cdots A$ & $D-\mathrm{H} \cdots A$ \\
\hline $\mathrm{N} 1-\mathrm{H} 1 \cdots \mathrm{O} 1$ & 0.88 & 1.86 & $2.739(3)$ & 175 \\
\hline
\end{tabular}

